INPLASY

PROTOCOL

To cite: Liao et al. The Effect of Mind-Body Exercise on the Pain Level of Patients with Cervical Pain: A protocol for Systemic Review and Metaanalysis. Inplasy protocol

202160050. doi:

10.37766/inplasy2021.6.0050

Received: 15 June 2021

Published: 15 June 2021

Corresponding author:

Xianhui Liao

liaoxianhui@whut.edu.cn

Author Affiliation:

Department of Sports, Wuhan

EQ\& IQ School.

Support:

2020GXNSFAA297115.

Review Stage at time of this submission: Data analysis.

Conflicts of interest:

None declared.

\section{The Effect of Mind-Body Exercise on the Pain Level of Patients with Cervical Pain: A protocol for Systemic Review and Meta-analysis}

Liao, $\mathrm{X}^{1} ; \mathrm{Ge}, \mathrm{B}^{2}$.

Review question / Objective: This study's aim is to explore the effect of mind-body exercise intervention on the pain level of patients with cervical pain.

Condition being studied: Cervical pain is a chronic disease. It is often caused in people who lower their heads for long periods. If the cervical pain cannot be relieved in time, it can easily lead to palpitations, insomnia, dizziness, etc., which can seriously affect people's physical and mental health. Pain is a relatively complex physical and psychological condition, and is the most common clinical symptom. It includes the feeling of pain caused by injury and stimulation to the body, and the body's pain response to noxious stimuli. Cervical pain is actually a chronic injury to the cervical spine. In order to reflect the degree of pain, the pain grade is often used to describe the degree of pain. In clinical description, the pain level is generally divided into 3 levels: mild, moderate, and severe pain. In order to achieve the purpose of the research, different studies will combine the actual situation of the research to digitally grade the pain, and achieve the effect of quantitatively distinguishing the grade of pain. In general, the level of pain can reflect the degree of discomfort.

INPLASY registration number: This protocol was registered with the International Platform of Registered Systematic Review and Meta-Analysis Protocols (INPLASY) on 15 June 2021 and was last updated on 15 June 2021 (registration number INPLASY202160050).

\section{INTRODUCTION}

Review question / Objective: This study's aim is to explore the effect of mind-body exercise intervention on the pain level of patients with cervical pain.
Condition being studied: Cervical pain is a chronic disease. It is often caused in people who lower their heads for long periods. If the cervical pain cannot be relieved in time, it can easily lead to palpitations, insomnia, dizziness, etc., which can seriously affect people's 
physical and mental health. Pain is a relatively complex physical and psychological condition, and is the most common clinical symptom. It includes the feeling of pain caused by injury and stimulation to the body, and the body's pain response to noxious stimuli. Cervical pain is actually a chronic injury to the cervical spine. In order to reflect the degree of pain, the pain grade is often used to describe the degree of pain. In clinical description, the pain level is generally divided into 3 levels: mild, moderate, and severe pain. In order to achieve the purpose of the research, different studies will combine the actual situation of the research to digitally grade the pain, and achieve the effect of quantitatively distinguishing the grade of pain. In general, the level of pain can reflect the degree of discomfort.

\section{METHODS}

Participant or population: Participants of this study are patients with cervical pain, who meet the pain diagnosis criteria are as follows: (1) "Practical Orthopedics" (4th edition); (2) "Expert Consensus on the Classification, Diagnosis and Non-Surgical Treatment of Cervical Spondylosis"; ( 3) "Guidelines for Diagnosis, Treatment and Rehabilitation of Cervical Spondylosis (2010)"; (4) Hospital admissions or selfassessment of neck pain; (5) "The Third National Cervical Spondylopathy Special Standards"; (6) "Clinical Diagnosis and Treatment Guidelines-Orthopedics Faculty"; (7) "Standards for the Second National Symposium on Cervical Spondylosis (1992)", etc.

Intervention: The study intervention is divided into two types: (1) the intervention group is a single mind-body exercise; (2) the intervention group has mind-body exercise and auxiliary measures.

Comparator: The study control is divided into two types: (1) auxiliary measures; (2) no intervention.

Study designs to be included: This study is a collection of peer-reviewed randomized controlled trials (RCTs).
Eligibility criteria: Inclusion criteria Types of study This study is a collection of peerreviewed randomized controlled trials (RCTs). Types of participants Participants of this study are patients with cervical pain, who meet the pain diagnosis criteria are as follows: (1) "Practical Orthopedics" (4th edition); (2) "Expert Consensus on the Classification, Diagnosis and Non-Surgical Treatment of Cervical Spondylosis"; ( 3) "Guidelines for Diagnosis, Treatment and Rehabilitation of Cervical Spondylosis (2010)"; (4) Hospital admissions or selfassessment of neck pain; (5) "The Third National Cervical Spondylopathy Special Standards"; (6) "Clinical Diagnosis and Treatment Guidelines-Orthopedics Faculty"; (7) "Standards for the Second National Symposium on Cervical Spondylosis (1992)", etc. Types of intervention The study intervention is divided into two types: (1) The intervention group is a single mind-body exercise (Taiji, Taijiquan, Baduanjin, Qigong, Wuqinxi, or Yijinjing), and the control group has no intervention or auxiliary measures (medicine, traction, acupuncture, massage, medium frequency electrotherapy, health exercise, static-contraction resistance training, Chinese medicine, health education, waiting list, exercise therapy, or cervical exercise. (2) The intervention group has mind-body exercise and auxiliary measures, or no intervention, and the control group has auxiliary measures or no intervention. Types of outcome measures Through a preliminary data search, the International Association for the Study of Pain defined: Pain is an unpleasant sensory experience, and an emotional experience accompanied by actual or potential tissue damage. Furthermore, this unpleasant sensory experience is often accompanied by the impact of related functions. The purpose of this study is to evaluate the effect of mind-body exercise intervention on the pain level of patients with cervical pain. Pain assessment: The more commonly used assessment scales in the world are the numerical rating scale (NRS), the VAS, and the McGill pain questionnaire (MPQ). The NRS, although widely used, it is not suitable for scientific research. The MPQ's evaluation items are more abundant, 
but the evaluation requires professionals and takes a long time. Combined with the characteristics of this study, the pain level of the population needs to be evaluated as soon as possible after the exercise intervention. Therefore, the pain level evaluation tool of this study is the VAS. Exclusion criteria (1) RCTs that are not published in peer-reviewed journals; (2) Mind-body exercise is not the main intervention; (3) Conference collections and documents of low academic level; (4) The experimental data cannot extract documents that cannot be calculated; (5) The participant has a history of headache or neck trauma; (6) Participants have received treatment before baseline measures.

Information sources: This study retrieved five databases, including CNKI (1979), Web of Science (1950), PubMed (1965), Cochrane (1991), and EBSCO (1949), date searched, May 10, 2021. This study has two keyword groups. Keyword group 1: mindbody exercise, Taiji, Taijiquan, Baduanjin, Qigong, Wuqinxi, and Yijinjing. Keyword group 2: cervical spondylosis, neck pain, and cervical pain, manually included, in accordance with the literature search strategy at the same time. The literature search was carried out simultaneously by the two authors to ensure the accuracy of the data search.

\section{Main outcome(s): VAS.}

Quality assessment / Risk of bias analysis: The two independent researchers used the modified Physiotherapy Evidence Database (PEDro) to evaluate the included articles and achieved independent evaluation results. If there was a difference in the evaluation results, they reached agreement through discussion. If the discussion failed to reach an agreement, the third researcher was invited to discuss and help solve it, and finally a consensus was reached.

Strategy of data synthesis: Subgroup and sensitivity analyses were used to explore the sources of heterogeneity in outcome indicators, combined effect size andpublication bias tests were used to calculate the effect size and risk of bias in the publications, and forest and funnel diagrams were drawn.

Subgroup analysis: Subgroup analyseswere used to explore the sources of heterogeneity in outcome indicators.

Sensitivity analysis: Sensitivity analyses were used to explore the sources of heterogeneity in outcome indicators.

Country(ies) involved: China.

Keywords: mind-body exercise, cervical pain, pain level, meta analysis.

Contributions of each author:

Author 1 - Xianhui Liao.

Author 2 - Beihai Ge. 